\title{
Modeling and Predicting Complex Patterns of Change Using Growth Component Models: An Application to Depression Trajectories in Cancer Patients
}

\author{
Axel Mayer \\ Friedrich Schiller University, Jena \\ Christian Geiser \\ Utah State University \\ Frank J. Infurna \\ German Institute for Economic Research (DIW Berlin), Berlin, Germany \\ Humboldt University Berlin, Germany \\ Christiane Fiege \\ Eberhard Carls University, Tübingen
}

This is a pre-copyedited, author-produced PDF of an article published in European Journal of Developmental Psychology following peer review.

\section{Citation:}

Mayer, A., Geiser, C. Infurna, F.J., \& Fiege, C. (2013). Modelling and predicting complex patterns of change using growth component models: An application to depression trajectories in cancer patients, European Journal of Developmental Psychology, 10, 40-59, doi:10.1080/17405629.2012.732721

Correspondence: Axel Mayer, Department of Methodology and Evaluation Research, Institute of Psychology, Friedrich Schiller University Jena, Am Steiger 3, Haus 1, 07743 Jena, Germany, Telephone: +49 3641 945236, E-mail: Axel.Mayer@uni-jena.de 


\section{Abstract}

In this paper, we present a general and flexible framework for constructively defining growth components to model complex change processes. Building on the concepts of the latent state-trait theory (LST theory; Steyer, Ferring, \& Schmitt, 1992), we develop structural equation models containing latent variables that represent latent growth (change) components of interest. We formulate these models based on an approach presented by Mayer, Steyer \& Mueller (in press). We discuss an application to the longitudinal course of depression in 2794 individuals from the Health and Retirement Study who experienced cancer diagnosis over the course of the study. We found that (1) on average, the depression trajectories showed a steep increase after diagnosis as well as an adaptation phase where levels returned back to levels prior to diagnosis, and (2) individual differences in change were large and could be partly explained by marital status and cognitive functioning.

Keywords: Growth Components, Multiple-Indicator Latent Growth Curve Models, True Change Models, Method Factors, Depression, Cancer diagnosis 


\section{Introduction}

The question of how to appropriately measure change over time using statistical methods is one of the most fundamental questions in developmental research, as development and change are at the heart of many theories in psychology and developmental science. Currently, conventional latent growth curve models (LGCMs; McArdle \& Epstein, 1987; Meredith \& Tisak, 1990) are among the most widely used statistical approaches for analyzing change over time in the social and behavioral sciences. Other popular models for the analysis of change are autoregressive models (e.g., Hertzog \& Nesselroade, 1987) and latent change score models (McArdle \& Hamagami, 2001; Raykov, 1999; Steyer, Eid, \& Schwenkmezger, 1997).

Most of these models can either be based on a single observed variable at each time point (single-indicator models) or on multiple observed variables at each time point (multiple-indicator models). Geiser, Keller, \& Lockhart (in press) and Leite (2007) have recently emphasized the advantages of using multiple indicator LGCMs.

In this paper, we present a multiple-indicator approach that facilitates the modeling of complex patterns of change. We illustrate the use of this approach based on an application to a longitudinal data set that deals with changes in depression in cancer patients before and after the patients received their cancer diagnosis (Infurna, Gerstorf, \& Ram, in press). We introduce a general and flexible framework for defining growth components based on a multistate model with method factors and apply the model to the depression trajectories of cancer patients.

\section{Modeling Complex Patterns of Change}


Conventional LGCMs are useful and widely applied models to analyze change across time. Application of these models can be complicated, however, when an investigator has to deal with complex, discontinuous patterns of change. In the linear LGCMs, for example, the growth trajectory is assumed to be linear for all individuals. Often, however, a researcher faces more complex growth trajectories. Even though more complex forms of change can in principle be represented in conventional LGCMs - either by specifying a more complex growth function (e.g., quadratic or cubic growth) or by freely estimating the loadings on the latent growth factor (McArdle \& Epstein, 1987) — the correct model specification for such processes is often complicated in the conventional LGCM framework. Multi-phase growth models (see Fauth, Gerstorf, Ram, \& Malmberg, 2012; Infurna, et al., in press, for details on this application) or piece-wise LGCMs (Bollen \& Curran, 2006) provide additional flexibility and can be used to model complex growth trajectories. Applications of these models can sometimes lack an explicit definition of latent variables, which can lead to difficulties in the interpretation of model parameters (see, e.g., Biesanz, Deeb-Sossa, Papadakis, Bollen, \& Curran, 2004; Rovine \& Molenaar, 1998; Schuster \& von Eye, 1998).

\section{The Longitudinal Course of Depression in Cancer Patients}

As an example of complex patterns of change, consider the means of depressive symptom scores in cancer patients as shown in Figure 1. There are five time points in this example. The first two measurements of depression took place four and two years before the patients received their cancer diagnosis (Occasion -4 and -2 in Figure 1). On the third time point (Occasion 0), patients had received their diagnosis within two years, and two additional follow-up measurements took place two and four years after the diagnosis (Occasions 2 and 4). In this case, change does not follow a straightforward continuous trend and cannot be appropriately modeled 
by a linear, quadratic, or cubic growth function. Even though the average depression score increased over time, there is a peak in the average depression score right after the diagnosis, followed by lower levels and less steep increase than was observed before the diagnosis.

Several questions are of interest to applied researchers in this context: "Are there individual differences in depression scores at baseline?", "Do depression scores increase over time, and are there individual differences in this increase?", "What effect does the cancer diagnosis have on depression scores?", and "(How) do persons adapt and what is the depression level after diagnosis?", "Is the growth process influenced by resilience factors, i.e., which external variables predict why some individuals adapt better to the diagnosis than others?" This follows from theoretical and conceptual models examining the extent to which mental health changes in relation to major life events, in this case, cancer diagnosis. Mental health changes in relation to major life events may proceed in stages or phases (Brickman \& Campbell, 1971; Diener, Lucas, \& Scollon, 2006). In a first phase, individuals react to the initial occurrence of the event, which may result in decrements in mental health. In a second phase, when some time has passed after an event (months to years), individuals' mental health may adapt or return back to their previous levels.

\section{The Growth Components Approach}

In this section, we show how complex patterns of growth as the one seen in cancer patients can be modeled in a theory-driven and straightforward way by defining latent growth components. The growth components approach (Mayer et al., in press) allows for a flexible modeling of change that can be tailored to the specific research question in a substantive application. In introducing our approach, we emphasize the advantages of defining the latent 
variables constructively on the basis of latent state-trait (LST) theory. The benefit of doing so is that we obtain a better understanding of what the growth components in the model represent, which allows us to clearly interpret all latent variables and coefficients included in the model.

\section{The Multistate Model With Method Factor}

The beginning point for the definition of growth components is the so-called multistate model (Steyer et al., 1992; Steyer, Schmitt, \& Eid, 1999) with measurement equivalence over time and a method factor (see Figure 2). It is important to establish measurement equivalence (time-invariant loadings and intercepts) in the multistate model before meaningful statements can be made about growth components, as changes in the metric of the latent state factors would preclude a sensible interpretation of growth processes in these factors (e.g., Ferrer, Balluerka \& Widaman, 2008). Method factors are used to account for indicator-specific components. In this article, we applied an approach to modeling these effects proposed by Pohl, Steyer, and Kraus (2008). In this approach, indicator-specific effects are modeled in terms of differences between latent state variables. Specifically for our applied example, we selected an indicator representing physical symptoms of depression as reference indicator (see Table 1 and the section about the empirical example for further information on the composition of the indicators). We defined a method effect variable for the second indicator (representing emotional symptoms of depression) as the difference between the latent state variable for emotional and the latent state variable for physical symptoms and made the assumption that the method effect variable is the same at each time point. The resulting multistate model with a method factor is shown in Figure 2.

\section{Constructing latent growth components}

Based on the multistate model, we can explicitly construct growth components that reflect the research questions raised in the section about the longitudinal course of depression in 
cancer patients. Growth components allow researchers to model true intra-individual change as well as inter-individual differences in these change components.

We follow Mayer et al.'s (in press) approach of first defining latent growth components and then deriving the corresponding path coefficients. The starting point is a model with five latent growth components $\pi_{j}(j=0,1, \ldots, 4)$ based on the latent state variables $\eta_{t}$. In the growth components approach, we need to specify as many growth components as latent state variables to obtain a saturated model. The latent growth components in our example are defined as follows:

$\pi_{0}=1 \cdot \eta_{1}$

$\pi_{1}=-2 \cdot \eta_{1}-1 \cdot \eta_{2}+1 \cdot \eta_{4}+2 \cdot \eta_{5}$

$\pi_{2}=-1 \cdot \eta_{1}-1 \cdot \eta_{2}+2 \cdot \eta_{3}$

$\pi_{3}=-1 \cdot \eta_{1}-1 \cdot \eta_{2}+1 \cdot \eta_{4}+1 \cdot \eta_{5}$

$\pi_{4}=1 \cdot \eta_{4}$,

resulting in the contrast matrix $\mathbf{C}$

$\mathbf{C}=\left(\begin{array}{ccccc}1 & 0 & 0 & 0 & 0 \\ -2 & -1 & 0 & 1 & 2 \\ -1 & -1 & 2 & 0 & 0 \\ -1 & -1 & 0 & 1 & 1 \\ 0 & 0 & 0 & 1 & 0\end{array}\right)$

The growth components approach requires a quadratic contrast matrix with full rank (i.e. without redundancies in the contrasts). The contrast matrix reveals the substantive meaning of the growth components:

Initial Level $\pi_{0}$. The values of the latent variable $\pi_{0}$ are the true scores of depression four years before the cancer diagnosis (i.e., the values on $\eta_{1}$ ). This variable serves as an intercept variable that reflects the initial true level of depression. 
Linear Change Component $\pi_{1}$. The linear change growth component $\pi_{1}$ represents linear change over time. $\pi_{1}$ plays a similar role as the slope factor in a multiple-indicator linear growth model by representing the linear trend across all five occasions of measurement.

Reaction Component $\pi_{2}$. This latent variable is a contrast between the average of the true scores of the first two occasions and the true scores measured up to two years following diagnosis. This growth component therefore reflects the reaction in depression after diagnosis. Higher scores on $\pi_{2}$ reflect a steeper increase after receiving the cancer diagnosis compared to the pre-diagnosis level.

Adaptation Component $\pi_{3}$. The values of $\pi_{3}$ are true difference scores of pre-diagnosis levels vs. post-diagnosis levels. A score of 0 on this variable would mean that the individual has the same average level of depression on the two occasions before diagnosis compared to the two occasions after diagnosis.

Post-Diagnosis Level $\pi_{4}$. The latent variable $\pi_{4}$ represents true depression scores two years after diagnosis (i.e., the values on $\eta_{4}$ ) and therefore reflects the post-diagnosis level of depression.

The C-matrix cannot directly be used in the structural part of a structural equation model (SEM) as in a SEM, the B-matrix of the structural model relates the latent variables to each other as follows: $\boldsymbol{\eta}=\mathbf{B} \boldsymbol{\pi}$. In contrast, the $\mathbf{C}$-matrix relates the latent variables as follows: $\boldsymbol{\pi}=\mathbf{C} \boldsymbol{\eta}$ (see Equation 1). In order to obtain the matrix of structural coefficients $\mathbf{B}$ that can be used in regular SEM, we need to solve Equation 1 for $\boldsymbol{\eta}$, i.e. we need to invert the $\mathbf{C}$-matrix (Mayer et al., in press):

$\mathbf{C}^{-1}=\mathbf{B}=\left(\begin{array}{ccccc}1 & 0 & 0 & 0 & 0 \\ 0 & 1 & 0 & -2 & 1 \\ 0.5 & 0.5 & 0.5 & -1 & 0.5 \\ 0 & 0 & 0 & 0 & 1 \\ 1 & 1 & 0 & -1 & 0\end{array}\right)$ 
The complete model including the method factor $M F$, the latent state variables $\eta_{t}$ and the growth components $\pi_{j}$ is shown in Figure 3. It can be seen that the coefficients in the inverted $\mathbf{C}$ matrix translate into structural (i.e., path) coefficients in the SEM. The growth component model implies that the latent growth components are a complete decomposition of the latent state factors. As a consequence, there are no residual variables at the level of the latent state factors. The scores on the latent state factors (and, consequently, also their variances and means) are fully determined by the latent growth components. All latent growth components have means and variances as model parameters to be estimated, reflecting average change as well as individual differences in change across time. Furthermore, all growth components can be correlated, as illustrated by the double-headed errors that connect these variables in Figure 3.

Given that the growth components represent a saturated decomposition of the latent state variables, the growth component model and the multistate model show an identical model fit. Nonetheless, the growth component version of the multistate model allows for more detailed insights regarding development and change across time.

\section{Empirical Application}

\section{Participants and procedure}

The Health and Retirement Study (HRS) is a nationally representative sample of households in the contiguous United States that consists of non-institutionalized adults aged 50 years and older $(N=30,000+$ individuals; for details, see McArdle et al., 2007; Soldo et al., 1997). Participants provide biennial reports on a wide range of economic, sociological, mental, and physical health measures. For the present analyses, we made use of data provided by those participants who (a) had not been diagnosed with a cancer at any point in their lives prior to the 
first occasion of measurement in 1992/1993, but were diagnosed with cancer for the first time at some point during their subsequent participation in the study (1994 to 2008; i.e., those who came into the study with a cancer diagnosis were not included in our analyses) and (b) provided data on all measures of interest. This subsample comprises $N=2794$ participants (age at diagnosis $M$ $=67.26, S D=9.86$, range $36-97 ; 47 \%$ women; $M=12$ years of education; $69 \%$ married). For further details on the sample, see Infurna et al. (in press).

Note that the primary goal of our paper was an empirical example of the demonstration of our method. It will be highly informative in future more mechanism-oriented studies to examine the extent to which differences in patterns of depressive symptoms change emerge amongst patients with various types of cancer and degree of severity.

\section{Measures}

Depressive symptoms. Eight items from the CES-D scale (Radloff, 1977) were used to assess depressive symptoms. The shorter scale with different response scale (yes/no versus several response categories ranging from "rarely or none of the time" to "most or all of the time") used in the HRS has demonstrated highly similar construct and external validity as the standard CESD (see Kohout, Berkman, Evants, \& Cornoni-Huntley, 1993). In order to illustrate the use of method factors in our model, we created two substantively heterogeneous item parcels. The first parcel ( $Y_{1 t}$ in our model) was composed of the physical symptom items, whereas the second parcel $\left(Y_{2 t}\right)$ comprised all emotional symptom items (see Table 1). The use of heterogeneous, but theory-based parcels allows one to examine whether the growth process is the same for different domains of a construct (here: physical vs. emotional symptoms of depression). We used the 
mean of the four items within each parcel as one indicator variable for our multiple-indicator SEMs. The means were scaled to a range from 0 to 10 .

Occasions: Time-to/from-cancer diagnosis. Cancer diagnosis was assessed at each wave using a single item, i.e., "Has a doctor ever told you that you have cancer or a malignant tumor, excluding minor skin cancer?". The onset of cancer diagnosis was defined as the wave of the first interview at which the participant reported being newly diagnosed with cancer. The number of years to and from this point of onset serves as the time-to/from-cancer diagnosis time metric. Realigning the time metric allowed us to define substantively meaningful occasions. We used five occasions of measurement: Four years before diagnosis, two years before diagnosis, within two years of diagnosis, two years after diagnosis and four years after diagnosis.

Predictors. The right side of Table 1 shows predictors that were hypothesized to influence changes in individuals' depressive symptoms in relation to cancer diagnosis. In terms of the resources individuals could draw from, we used the reports obtained at the wave prior to cancer diagnosis. These included age, gender, years of education, marital status, memory, self-rated health and functional limitations. Memory was measured as the proportion of words correctly remembered from both the immediate and delayed free-recall tests, i.e., 20 words in total, with higher scores representing better memory (McArdle et al., 2007). Self-rated health was measured with a single item asking whether their health is excellent, very good, good, fair or poor. Functional limitations were measured using a composite sum index of the number of everyday activities participants reported having any difficulty completing. Higher scores represent greater functional limitations or poorer physical functioning (Rodgers \& Miller, 1997).

\section{Results}


First, we fit a multistate model with time-invariant factor loadings and intercepts and a method factor as shown in Figure 2. We used the physical and emotional symptoms parcels as the two indicator variables per occasion. We fit the model using the robust maximum likelihood (MLR) estimator in Mplus 6.1 (Muthén \& Muthén, 1998-2010). The multistate model with method factor (see Figure 2) showed an adequate fit, $\chi^{2}(28, N=2794)=87.979, p<.01$; CFI $=$ $.989 ; \mathrm{RMSEA}=.027 ; \mathrm{SRMR}=.021$. Detailed results for this model are shown in Table 2. Descriptively, the means of the latent state variables increased over time [from $M\left(\eta_{1}\right)=2.25$ to $\left.M\left(\eta_{5}\right)=2.82\right]$, and there was a steep increase in means from the pre-diagnosis occasion two years before diagnosis to the diagnosis occasion. There were considerable inter-individual differences in the latent depression scores especially two and four years after the diagnosis occasion, which was reflected by the variances of the latent state variables. The mean of the method factor was negative $[M(M F)=-0.96]$ indicating that on average, participants scored higher on the physical symptoms than on the emotional symptoms parcel. The variance of the method factor $[\operatorname{Var}(M F)$ $=2.00 \mathrm{]}$ reflects inter-individual differences with respect to the size of the method effect. That is, for some individuals there was a larger difference between the physical and emotional symptom scores than for others. Not only the size of this difference varied across individuals, but also whether the method effect was positive or negative (i.e., for some individuals this difference was negative, whereas for others it was positive).

\section{Latent Growth Components}

In the next step, we added the growth components to the multistate model with method factors to obtain more detailed information about changes in depression across time. Recall that we defined five growth components: Initial level $\pi_{0}$, linear change component $\pi_{1}$, reaction 
component $\pi_{2}$, adaptation component $\pi_{3}$, and post-diagnosis level $\pi_{4}$. We used the B-matrix derived in Equation 3 to decompose the latent state variables $\eta_{t}$ into growth components $\pi_{j}$. The complete model is shown in Figure 3. Since the growth components represent a saturated decomposition of the latent state variables, the growth component model and the multistate model with method factor have the same model fit. The estimated means and variances of the growth components are shown in Table 3. Note that the means and variances of the initial level $\pi_{0}$ and the post-diagnosis level $\pi_{4}$ are identical with the corresponding parameters of the latent state variables $\eta_{1}$ and $\eta_{4}$ in the multistate model with method factors, because we defined these growth components to be identical with the initial and post-diagnoses levels, respectively (see Equation 1).

The mean of the linear growth component was $M\left(\pi_{1}\right)=1.56(S E=0.15)$ indicating an increase in average depression scores over time. There were also noticeable inter-individual differences on this growth components $\left[\operatorname{Var}\left(\pi_{1}\right)=19.92\right]$. The linear growth component ignores the critical life event "cancer diagnosis", and its values represent a linear approximation for every person's change in depression scores over time.

We hypothesized a steep increase in depression scores in reaction to the cancer diagnosis. This hypothesis was reflected by the reaction growth component $\pi_{2}$. Its mean was $M\left(\pi_{2}\right)=0.92$ $(S E=0.10)$. In order to calculate the average increase of depression scores in reaction to the diagnoses, we have to divide 0.92 by two $(0.92 / 2=0.46)$. This is because the growth component $\pi_{2}$ has been defined in such a way that the sum of the depression scores of the two pre-diagnosis occasions is compared to two times the score at the diagnosis occasion (see Equation 1). Hence, participants, on average, demonstrated a 0.46 point increase on the depression scale in reaction to their diagnosis. However, persons reacted very differently, as indicated by the rather large 
variance estimate for $\pi_{2}\left[\operatorname{Var}\left(\pi_{2}\right)=12.84\right]$. While some persons experienced a steep increase in depression scores, others even showed a decline in their depression scores after receiving the diagnosis.

As mentioned previously, change in depression scores after cancer diagnosis may proceed in two phases. After the initial reaction phase, there may be an adaptation phase, in which people's depression scores may decline again, or at least show a less steep increase compared to the time immediately following the diagnosis. The adaptation phase is reflected in the growth component $\pi_{3}$. Its mean is $M\left(\pi_{3}\right)=0.99(S E=0.09)$, indicating that average depression was still elevated at the two post-diagnosis occasions compared to the two prediagnosis occasions (on average by 0.99 points on the depression scale). We also found considerable inter-individual differences in the adaptation growth component $\left[\operatorname{Var}\left(\pi_{3}\right)=8.17\right]$. Part of this heterogeneity may be due to person characteristics, differences in health and/or available resources. We further examined these hypotheses by including predictors of growth components (see next section).

The post-diagnosis level was represented by the growth component $\pi_{4}$. Its mean was $M\left(\pi_{4}\right)=2.72(S E=0.06)$, showing an elevated level of depression relative to the initial state $\left[M\left(\pi_{0}\right)=2.25, S E=0.06\right]$

\section{Predictors of Growth Components}

Typically, investigators are not only interested in describing patterns of change, but also in predictors that may explain certain aspects of individual differences in change. Table 1 lists the predictors that we judged to be substantively interesting in the context of change in depression after cancer. We included all of these predictors in the complete model by 
simultaneously estimating the regression of all five growth components $\pi_{j}$ on all of the predictors $Z_{1}-Z_{7}$ :

$E\left(\pi_{j} \mid \boldsymbol{Z}\right)=\gamma_{0 j}+\gamma_{1 j} Z_{1}+\ldots+\gamma_{7 j} Z_{7}$.

Appendices A and B provide the Mplus (Muthén \& Muthén, 1998-2010) and lavaan (Rosseel, in press) scripts for the complete models including the regressions of the growth components on predictors. Table 4 shows the results for all regression analyses.

We focus on the role of marital status and memory, since these two predictors show consistent effects on the growth components that represent change in depression, i.e., $\pi_{1}-\pi_{3}$ (see Table 4). Note that all of the coefficients reported in Table 4 represent partial regression coefficients that capture the effects of one predictor on each of the growth component while controlling for all other predictors. We hypothesized that marital status would serve as a proxy for available resources, leading to better adaptation and therefore a reduction in the increase in depression scores after diagnosis. In contrast to our expectation, we found that - on average married persons showed a steeper overall linear slope in depression over time $\left(\gamma_{71}=1.10, p<\right.$ $.01)$, that they had a greater increase of depression under diagnosis $\left(\gamma_{72}=0.84, p<.01\right)$, and that they adapted less well $\left(\gamma_{73}=0.72, p<.01\right)$. Note, however, that married persons had lower levels of depression four years prior to their diagnosis $\left(\gamma_{70}=-0.71, p<.01\right)$ and still had lower levels of depression at the occasion two years after diagnosis $\left(\gamma_{74}=-0.28, p=.02\right)$.

With regard to memory, we found that persons with better memory showed a less steep linear slope in depression over time $\left(\gamma_{51}=-3.21, p<.01\right)$, a lower increase in depression under diagnosis $\left(\gamma_{52}=-1.20, p=.04\right)$, and better adaptation $\left(\gamma_{53}=-2.01, p<.01\right)$. While memory had no significant effect on depression four years prior to diagnosis $\left(\gamma_{50}=-0.28, p=.32\right)$, there was a negative effect of memory on depression two years after receiving cancer diagnosis $\left(\gamma_{54}=-1.75\right.$, 
$p<.01)$. Again, interpretation of regression coefficients is on average, controlling for all other predictors in the model. Assuming that memory serves as a proxy for cognitive resources, we conclude that cognitive resources seem to have a protective effect.

\section{Discussion}

In this article, we showed how to define latent growth components on the basis of welldefined latent state variables obtained from LST theory. Formulating latent growth models on the basis of well-defined latent variables has the advantage that the meaning of the growth components and the underlying theoretical assumptions of the model become clearer. These growth components can represent complex patterns of change and offer researchers a flexible way to modeling their specific substantive research questions.

\section{Most Important Results}

In our data example, we applied the growth components approach to analyze changes in latent depression scores in cancer patients before and after the patients had received their cancer diagnosis. Different growth components were needed to properly account for (1) overall linear changes in depression over time, (2) specific increases in depressive symptoms after receiving the diagnosis, and (3) long-term adaptation effects. On average, we found that (1) there was an increase in depression scores over time, that (2) there was a steep increase in average depression scores as a reaction to the cancer diagnosis, and that (3) even though there was partial adaptation, average levels of depression were still elevated four years after diagnosis. Of note, there were considerable inter-individual differences in all of the growth components. We therefore also examined the role of potential risk factors and protective factors by including them in the model as predictors of the growth components. Surprisingly, we found marital status to 
have negative effects on change in depression scores. This may be due to increased worries about the partner and the family. Also, persons who were in a relationship had lower depression scores at baseline. Maybe there is just more room for increases in depression in these individuals compared to unmarried persons. For memory, we consistently found positive effects on the growth components that represented change. Assuming that memory serves as a proxy for cognitive resources, we conclude that cognitive resources seem to have a protective effect with regard to depression in cancer patients.

\section{Comparisons of Approaches to Modeling Change}

Growth component models (Mayer et al., in press) are saturated latent change models. In contrast to existing approaches, the growth component approach allows researchers to define specific components of change in a theory-driven way. The particular strength of this approach is that researchers can explicitly define the latent variables according to their substantive research questions. Growth component models are multiple-indicator models and are based on LST theory.

Conventional latent growth curve models such as linear and quadratic LGCMs are currently among the most popular statistical approaches for measuring change over time. These models are typically formulated as single-indicator models and assume a specific functional form of average and individual change over time. Conventional LGCMs require strong assumptions that are often violated in social science data, and the interpretation of parameters can be difficult. Using conventional LGCMs, it is difficult to model complex patterns of change, especially when change is not continuous, as in our example with change in depression scores after cancer diagnosis. 
LGCMs with freely estimated loadings (McArdle \& Epstein, 1987) relax the requirement of a pre-specified functional form of development. These models allow estimating the functional form of change from the data. However, LGCMs with freely estimated loadings are not as theory-driven as the growth components approach and they are not saturated, i.e., it is assumed in these models that all individuals change in a particular manner.

Another class of SEMs which does not require predefined trajectories are latent change models. Examples of such models include baseline and neighbor true change models (Steyer et al., 1997), latent change score models aka latent difference score models (McArdle \& Hamagami, 2001), and the models proposed by Raykov (1999). These models are typically used to model true changes between adjacent measurement occasions or change relative to a baseline occasion. Like the growth components models presented here, latent change models typically are specified to be saturated at the level of the latent state variables. Therefore, they will show an identical fit as the models presented here when fit to the same data. Latent change models can also be specified to test specific theory-derived components of change. However, specific hypothesis about different components of change can be tested in a more explicit and elegant way with the growth components approach.

So-called piece-wise growth models and multi-phase growth models can also deal with complex patterns of change. These models are usually formulated as single-indicator models, even though they can in principle be extended to the multiple-indicator case. Latent variables in these models are typically not explicitly defined based on LST theory, which can make the meaning of resulting parameters less clear than in the growth components approach. See Infurna et al. (in press) for an application of a multi-phase growth model to the same data set as used in this article. Note that, even though the specification of the growth component model and the 
multi-phase model are very different, the two approaches yielded similar results for this particular empirical example.

In this paper, we defined growth components for complex patterns of change based on latent state variables. According to LST theory, a latent state variable contains both, a latent trait and a latent state residual component. Even though the trait component characterizes personspecific effects only, it may change systematically over time. Eid and Hoffmann (1998) showed that latent change and latent growth curve models can also be formulated at the level of latent trait rather than latent state variables leading to latent trait change models. An advantage of latent trait change models is that situation-specific fluctuations and person $\times$ situation interaction effects can be separated from latent trait effects and true latent trait change, which is not possible in the growth component approach presented in this paper. 


\section{References}

Biesanz, J. C., Deeb-Sossa, N., Papadakis, A. A., Bollen, K. A., \& Curran, P. J. (2004). The role of coding time in estimating and interpreting growth curve models. Psychological Methods, 9 (1), 30-52.

Bollen, K. A., \& Curran, P. J. (2006). Latent curve models: A structural equation approach. Hoboken, NJ: Wiley.

Brickman, P., \& Campbell, D. T. (1971). Hedonic relativism and planning the good society. In M. H. Appley (Ed.), Adaptation level theory: A symposium (pp. 287-302). New York, Academic Press.

Diener, E., Lucas, R. E., \& Scollon, C. N. (2006). Beyond the hedonic treadmill: Revising the adaptation theory of well-being. American Psychologist, 61, 305-314.

Eid, M., \& Hoffmann, L. (1998). Measuring variability and change with an item response model for polytomous variables. Journal of Educational and Behavioral Statistics, $23,193-215$.

Fauth, E. B., Gerstorf, D., Ram, N., \& Malmberg, B. (2012). Changes in depressive symptoms in the context of disablement processes: Role of demographic characteristics, cognitive function, health, and social support. Journals of Gerontology, Series B: Psychological Sciences and Social Sciences, 67, 167-177.

Ferrer, E., Balluerka, N., \& Widaman, K. F. (2008). Factorial invariance and the specification of second-order growth models. methodology, 4, 22-36. Methodology, 4, 22-36. 
Geiser, C., Keller, B. T., \& Lockhart, G. (in press). First versus second order latent growth curve models: Some insights from latent state-trait theory. Structural Equation Modeling.

Hertzog, C., \& Nesselroade, J. R. (1987). Beyond autoregressive models: Some implications of the trait-state distinction for the structural modeling of developmental change. Child Development, 58 (1), 93-109.

Infurna, F. J., Gerstorf, D., \& Ram, N. (in press). The nature and correlates of change in depressive symptoms with cancer diagnosis: Reaction and adaptation. Psychology and Aging.

Kohout, F. J., Berkman, L. F., Evans, D. A. \& Cornoni-Huntley, J. (1993). Two shorter forms of the CES-D depression symptoms index. Journal of Aging and Health, 5, 179-193.

Leite, W. L. (2007). A comparison of latent growth models for constructs measured by multiple items. Structural Equation Modeling, 14, 581-610.

Mayer, A., Steyer, R., \& Mueller, H. (in press). A general approach to defining latent growth components. Structural Equation Modeling.

McArdle, J. J., \& Epstein, D. (1987). Latent growth curves within developmental structural equation models. Child Development, 58, 110-133.

McArdle, J. J., Fisher, G. G., \& Kadlec, K. M. (2007). Latent variable analyses of age trends from the health and retirement study, 1992-2004. Psychology and Aging, 22, $525-545$.

McArdle, J. J., \& Hamagami, F. (2001). Latent difference score structural models for linear dynamic analyses with incomplete longitudinal data. In L. M. Collins \&

A. G. Sayer (Eds.), New methods for the analysis of change (pp. 139-175). 
Washington, DC: American Psychological Association.

Meredith, M., \& Tisak, J. (1990). Latent curve analysis. Psychometrika, 55, 107-122.

Mor, V., Allen, S., \& Malin, M. (1994). The psychosocial impact of cancer on older vs. younger patients and their families. Cancer, 74, 2118-2127.

Muthén, L. K., \& Muthén, B. O. (1998-2010). Mplus User's Guide (6th ed.) [Computer software manual]. Los Angeles, CA: Muthén and Muthén.

Pohl, S., Steyer, R., \& Kraus, K. (2008). Modelling method effects as individual causal effects. Journal of the Royal Statistical Society: Series A, 171, 41-63.

Radloff, L. S. (1977). The CES-D Scale: A self-report depression scale for research in the general population. Applied Psychological Measurement, 1, 385-401.

Raykov, T. (1999). Are simple change scores obsolete? An approach to studying correlates and predictors of change. Applied Psychological Measurement, 23, 120-126.

Rodgers, W. L., \& Miller, B. (1997). A comparative analysis of ADL questions in surveys of older people. The Journals of Gerontology: Psychological Sciences, 52B, 21-36.

Rosseel, Y. (in press). lavaan: An R package for structural equation modeling. Journal of Statistical Software.

Rovine, M. J., \& Molenaar, P. (1998). The covariance between level and shape in the latent growth curve model with estimated basis vector coefficients. Methods of Psychological Research Online, 3(2).

Schuster, C., \& von Eye, A. (1998). Determining the meaning of parameters in multilevel models for longitudinal data. International Journal of Behavioral Development, $22(3), 475-491$.

Soldo, B., Hurd, M., Rodgers, W., \& Wallace, R. (1997). Asset and health dynamics 
among the oldest old: An overview of the AHEAD study. Journals of Gerontology: Psychological Sciences and Social Sciences, 52B, 1-20.

Steyer, R., Eid, M., \& Schwenkmezger, P. (1997). Modeling true intraindividual change: True change as a latent variable. Methods of Psychological Research Online, 2, $21-33$.

Steyer, R., Ferring, D., \& Schmitt, M. J. (1992). States and traits in psychological assessment. European Journal of Psychological Assessment, 8, 79-98.

Steyer, R., Schmitt, M., \& Eid, M. (1999). Latent state-trait theory and research in personality and individual differences. European Journal of Personality, 13, 389-408. 
Table 1

Overview of Variables

\begin{tabular}{lll}
\hline Parcel 1 (Physical Symptoms) & Parcel 2 (Emotional & Predictors \\
& Symptoms) & \\
\hline Felt Depressed & Happy (Reverse Coded) & Age \\
Effort & Lonely & Gender \\
Sleep & Enjoy Life (Reverse Coded) & Education \\
Not get going & Sad & Marital Status \\
& & Memory \\
& & Functional limitations
\end{tabular}


Table 2

Results for the Multistate Model with Method Factor

\begin{tabular}{|c|c|c|c|c|c|}
\hline & Estimate & $S E$ & & Estimate & $S E$ \\
\hline Means & & & Residual V & ices & \\
\hline$M\left(\eta_{1}\right)$ & 2.25 & 0.06 & $\operatorname{Var}\left(\varepsilon_{11}\right)$ & 2.87 & 0.19 \\
\hline$M\left(\eta_{2}\right)$ & 2.31 & 0.05 & $\operatorname{Var}\left(\varepsilon_{21}\right)$ & 2.19 & 0.18 \\
\hline$M\left(\eta_{3}\right)$ & 2.74 & 0.06 & $\operatorname{Var}\left(\varepsilon_{12}\right)$ & 2.68 & 0.16 \\
\hline$M\left(\eta_{4}\right)$ & 2.72 & 0.06 & $\operatorname{Var}\left(\varepsilon_{22}\right)$ & 1.78 & 0.15 \\
\hline$M\left(\eta_{5}\right)$ & 2.82 & 0.07 & $\operatorname{Var}\left(\varepsilon_{13}\right)$ & 3.21 & 0.18 \\
\hline$M(M F)$ & -0.96 & 0.04 & $\operatorname{Var}\left(\varepsilon_{23}\right)$ & 2.32 & 0.16 \\
\hline Variances & & & $\operatorname{Var}\left(\varepsilon_{14}\right)$ & 3.15 & 0.20 \\
\hline $\operatorname{Var}\left(\eta_{1}\right)$ & 2.98 & 0.20 & $\operatorname{Var}\left(\varepsilon_{24}\right)$ & 2.98 & 0.20 \\
\hline $\operatorname{Var}\left(\eta_{2}\right)$ & 2.96 & 0.21 & $\operatorname{Var}\left(\varepsilon_{15}\right)$ & 2.96 & 0.21 \\
\hline $\operatorname{Var}\left(\eta_{3}\right)$ & 2.44 & 0.19 & $\operatorname{Var}\left(\varepsilon_{25}\right)$ & 2.44 & 0.19 \\
\hline $\operatorname{Var}\left(\eta_{4}\right)$ & 6.27 & 0.28 & Model Fit & & \\
\hline $\operatorname{Var}\left(\eta_{5}\right)$ & 6.24 & 0.31 & $\chi^{2}(28)$ & 87.979 & \\
\hline $\operatorname{Var}(M F)$ & 2.00 & 0.11 & RMSEA & 0.027 & \\
\hline
\end{tabular}

Note. Covariances between all latent variables are not shown but have been included. 
Table 3

Means and Variances of Latent Growth Components

\begin{tabular}{llllll}
\hline & Estimate & SE & & Estimate & SE \\
\hline $\mathrm{M}\left(\pi_{0}\right)$ & 2.25 & 0.06 & $\operatorname{Var}\left(\pi_{0}\right)$ & 5.32 & 0.26 \\
$\mathrm{M}\left(\pi_{1}\right)$ & 1.56 & 0.15 & $\operatorname{Var}\left(\pi_{1}\right)$ & 19.92 & 1.79 \\
$\mathrm{M}\left(\pi_{2}\right)$ & 0.92 & 0.10 & $\operatorname{Var}\left(\pi_{2}\right)$ & 12.84 & 0.90 \\
$\mathrm{M}\left(\pi_{3}\right)$ & 0.99 & 0.09 & $\operatorname{Var}\left(\pi_{3}\right)$ & 8.17 & 0.68 \\
$\mathrm{M}\left(\pi_{4}\right)$ & 2.72 & 0.06 & $\operatorname{Var}\left(\pi_{4}\right)$ & 6.27 & 0.28 \\
\hline
\end{tabular}


Table 4

Regressions with Predictors

$E\left(\pi_{0} \mid Z\right) \quad E\left(\pi_{1} \mid Z\right) \quad E\left(\pi_{2} \mid Z\right) \quad E\left(\pi_{3} \mid Z\right) \quad E\left(\pi_{4} \mid Z\right)$

\begin{tabular}{lcccccccccc}
\hline & Estimate & $S E$ & Estimate & $S E$ & Estimate & $S E$ & Estimate & $S E$ & Estimate & $S E$ \\
Education & $-0.09^{*}$ & 0.02 & -0.01 & 0.06 & -0.05 & 0.03 & -0.02 & 0.03 & $-0.11^{*}$ & 0.02 \\
Women & 0.11 & 0.10 & 0.07 & 0.31 & 0.24 & 0.21 & 0.02 & 0.19 & 0.20 & 0.11 \\
Age & $-0.03^{*}$ & 0.01 & 0.03 & 0.02 & -0.01 & 0.01 & 0.01 & 0.01 & $-0.02^{*}$ & 0.01 \\
Functional & $0.38^{*}$ & 0.05 & -0.25 & 0.15 & -0.11 & 0.09 & $-0.18^{*}$ & 0.09 & $0.32^{*}$ & 0.05 \\
Limitations & & & & & & & & & & \\
Memory & -0.28 & 0.29 & $-3.21^{*}$ & 0.90 & $-1.20 *$ & 0.59 & $-2.01 *$ & 0.55 & $-1.75^{*}$ & 0.32 \\
Self-rated Health & $-0.49^{*}$ & 0.05 & -0.24 & 0.16 & 0.00 & 0.11 & -0.14 & 0.10 & $-0.52^{*}$ & 0.06 \\
Married/Partnered & $-0.71 *$ & 0.12 & $1.10^{*}$ & 0.36 & $0.84^{*}$ & 0.23 & $0.72 *$ & 0.22 & $-0.28^{*}$ & 0.12 \\
\hline
\end{tabular}

Note. ${ }^{*} p<.05$. 
Figure 1. Fictive Individual and Mean Trajectories

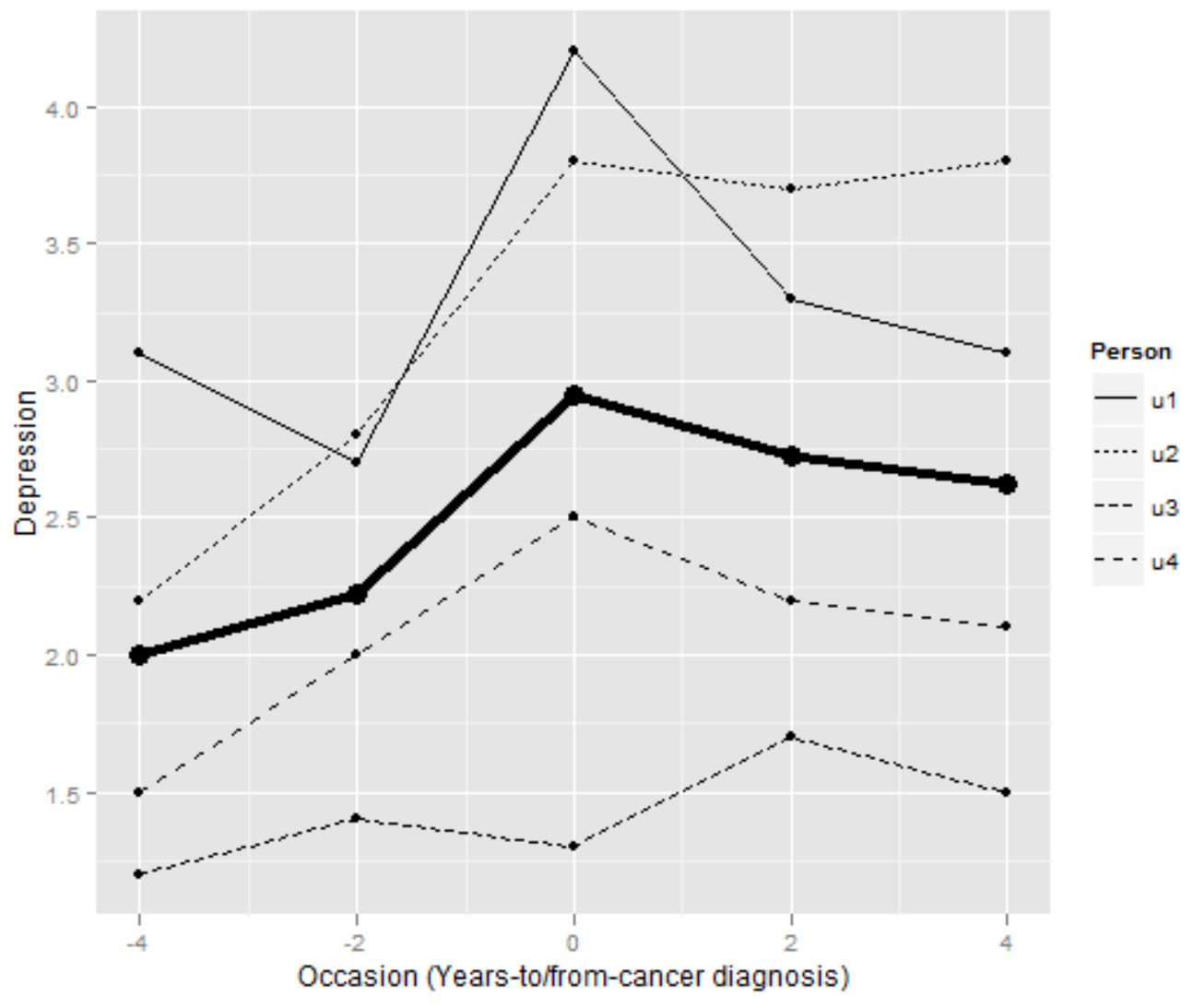


Figure 2. Multistate model with method factor.

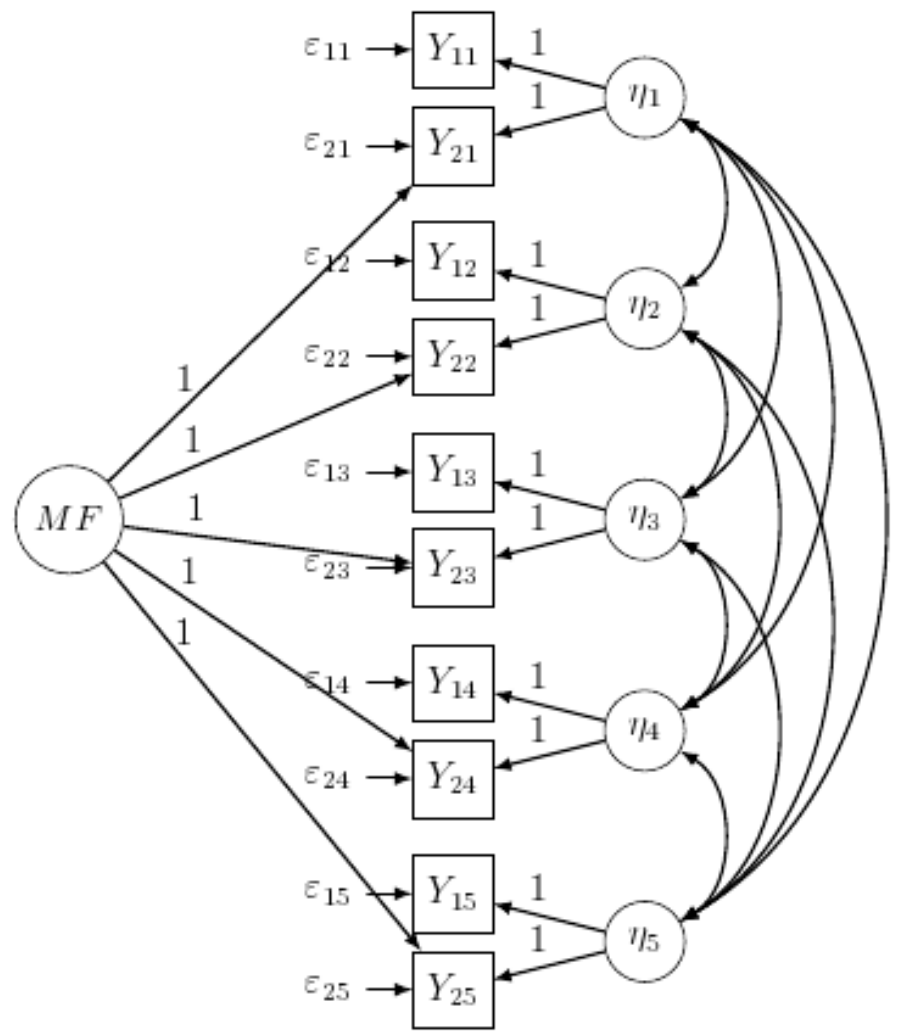


Figure 3. Complete model with latent state variables $\eta_{t}$, growth components $\pi_{j}$ and method factor $M F$.

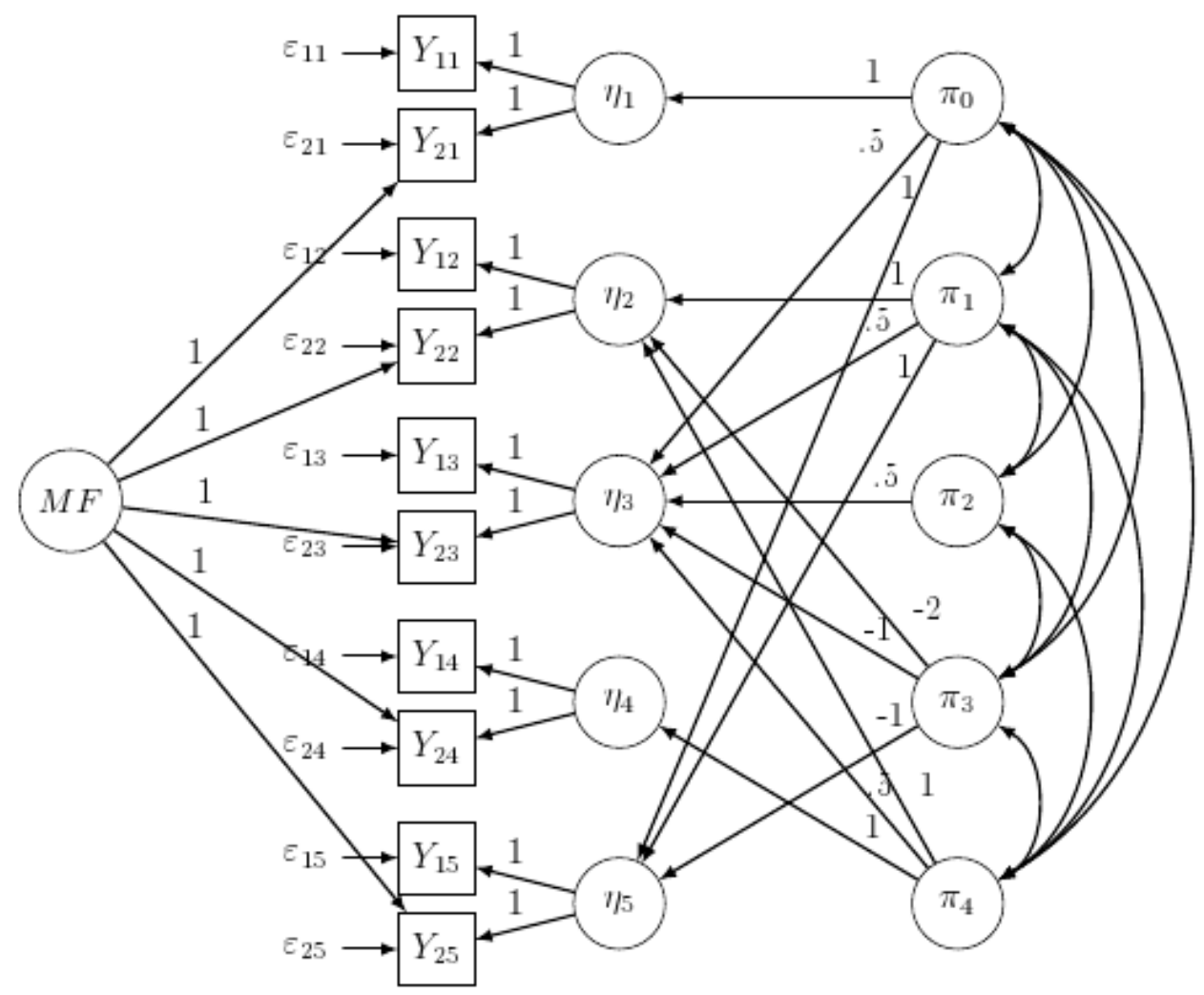




\section{Appendix A}

\section{Mplus Input}

TITLE: Latent Growth Components with Predictors

ANALYSIS: ESTIMATOR = MLR;

MODEL: ! Measurement Model for etas

eta1byy11@1 y21@1;

eta2 byy12@1 y22@1;

eta3byy13@1y23@1;

eta4byy14@1y24@1;

eta5byy15@1 y25@1;

! Method Factor

mf by y21@1 y22@1 y23@1 y24@1 y25@1;

[mf*];

[y11@0y12@0y13@0y14@0y15@0];

[y21@0y22@0y23@0y24@0y25@0];

[eta1-eta5@0];

eta1-eta5@0;

!Growth Components

pi0by eta1@1 eta3@0.5 eta5@1;

pi1 by eta2@1 eta3@0.5 eta5@1;

pi2 by eta3@0.5;

pi3by eta2@-2 eta3@-1 eta5@-1;

pi4 by eta2@1 eta3@0.5 eta4@1;

[pi0-pi4*];

! Regression on Predictors

pi0 on educ gender age adl rec srh married;

pil on educ gender age adl rec srh married;

pi 2 on educ gender age adl rec srh married;

pi3 on educ gender age adl rec srh married;

pi4 on educ gender age adl rec srh married; 


\section{Appendix B}

\section{lavaan Input}

We also provide lavaan 0.4-13 (Rosseel, in press) input for the complete model.

$$
\begin{aligned}
& \text { eta } 1=\sim 1 * \mathrm{y} 11+1 * \mathrm{y} 21 \\
& \text { eta } 2=\sim 1 * \mathrm{y} 12+1 * \mathrm{y} 22 \\
& \text { eta3 }=\sim 1 * \mathrm{y} 13+1 * \mathrm{y} 23 \\
& \text { eta } 4=\sim 1 * \mathrm{y} 14+1 * \mathrm{y} 24 \\
& \text { eta5 }=\sim 1 * \mathrm{y} 15+1 * \mathrm{y} 25 \\
& \mathrm{mf}=\sim 1 * \mathrm{y} 21+1 * \mathrm{y} 22+1 * \mathrm{y} 23+1 * \mathrm{y} 24+1 * \mathrm{y} 25 \\
& \mathrm{y} 11+\mathrm{y} 12+\mathrm{y} 13+\mathrm{y} 14+\mathrm{y} 15 \sim 0 * 1 \\
& \mathrm{y} 21+\mathrm{y} 22+\mathrm{y} 23+\mathrm{y} 24+\mathrm{y} 25 \sim 0 * 1 \\
& \text { eta } 1+\text { eta } 2+\text { eta } 3+\text { eta } 4+\text { eta } 5 \sim 0 * 1 \\
& \mathrm{mf} \sim 1 \\
& \text { eta1 } \sim 0 * \text { eta } 1 \\
& \text { eta2 } \sim 0 * \text { eta2 } \\
& \text { eta3 } \sim 0 * \text { eta3 } \\
& \text { eta } 4 \sim 0 * \text { eta } 4 \\
& \text { eta5 } \sim 0 * \text { eta5 } \\
& \text { pi } 0=\sim 1 * \text { eta } 1+0.5 * \text { eta } 3+1 * \text { eta } 5 \\
& \text { pi } 1=\sim 1 * \text { eta } 2+0.5 * \text { eta } 3+1 * \text { eta } 5 \\
& \text { pi2 }=\sim 0.5 * \text { eta } 3 \\
& \text { pi3 }=\sim-2 * \text { eta } 2+-1 * \text { eta } 3+-1 * \text { eta5 } \\
& \text { pi } 4=\sim 1 * \text { eta } 2+0.5 * \text { eta } 3+1 * \text { eta } 4 \\
& \mathrm{pi} 0+\text { pi } 1+\text { pi } 2+\text { pi } 3+\text { pi } 4 \sim 1 \\
& \text { pi } 0 \sim \text { educ }+ \text { gender }+ \text { age }+ \text { adl }+ \text { rec }+ \text { srh }+ \text { married } \\
& \text { pi } 1 \sim \text { educ }+ \text { gender }+ \text { age }+ \text { adl }+ \text { rec }+ \text { srh }+ \text { married } \\
& \text { pi } 2 \sim \text { educ }+ \text { gender }+ \text { age }+ \text { adl }+ \text { rec }+ \text { srh }+ \text { married } \\
& \text { pi } 3 \sim \text { educ }+ \text { gender }+ \text { age }+ \text { adl }+ \text { rec }+ \text { srh }+ \text { married } \\
& \text { pi } 4 \sim \text { educ }+ \text { gender }+ \text { age }+ \text { adl }+ \text { rec }+ \text { srh }+ \text { married }
\end{aligned}
$$


Erratum to: Mayer, A., Geiser, C., Infurna, F. J., \& Fiege, C. (2013). Modelling and predicting complex patterns of change using growth component models: An application to depression trajectories in cancer patients. European Journal of Developmental Psychology, 10, 40-59.

There was a typo in the published version of Table 2. The estimates and standard errors for $\operatorname{Var}\left(\eta_{1}\right), \operatorname{Var}\left(\eta_{2}\right)$, and $\operatorname{Var}\left(\eta_{3}\right)$ were copied and pasted from the right-hand column, and by this mistake, they corresponded to variances of measurement error variables. Please see the corrected Table 2 (below) for the correct estimates and standard errors.

Table 2 (corrected). Results for the Multistate Model with Method Factor

\begin{tabular}{|c|c|c|c|c|c|}
\hline & Estimate & $S E$ & & Estimate & $S E$ \\
\hline Means & & & Residual & aces & \\
\hline$M\left(\eta_{1}\right)$ & 2.25 & 0.06 & $\operatorname{Var}\left(\varepsilon_{11}\right)$ & 2.87 & 0.19 \\
\hline$M\left(\eta_{2}\right)$ & 2.31 & 0.05 & $\operatorname{Var}\left(\varepsilon_{21}\right)$ & 2.19 & 0.18 \\
\hline$M\left(\eta_{3}\right)$ & 2.74 & 0.06 & $\operatorname{Var}\left(\varepsilon_{12}\right)$ & 2.68 & 0.16 \\
\hline$M\left(\eta_{4}\right)$ & 2.72 & 0.06 & $\operatorname{Var}\left(\varepsilon_{22}\right)$ & 1.78 & 0.15 \\
\hline$M\left(\eta_{5}\right)$ & 2.82 & 0.07 & $\operatorname{Var}\left(\varepsilon_{13}\right)$ & 3.21 & 0.18 \\
\hline$M(M F)$ & -0.96 & 0.04 & $\operatorname{Var}\left(\varepsilon_{23}\right)$ & 2.32 & 0.16 \\
\hline Variances & & & $\operatorname{Var}\left(\varepsilon_{14}\right)$ & 3.15 & 0.20 \\
\hline $\operatorname{Var}\left(\eta_{1}\right)$ & 5.32 & 0.26 & $\operatorname{Var}\left(\varepsilon_{24}\right)$ & 2.98 & 0.20 \\
\hline $\operatorname{Var}\left(\eta_{2}\right)$ & 6.05 & 0.25 & $\operatorname{Var}\left(\varepsilon_{15}\right)$ & 2.96 & 0.21 \\
\hline $\operatorname{Var}\left(\eta_{3}\right)$ & 6.98 & 0.27 & $\operatorname{Var}\left(\varepsilon_{25}\right)$ & 2.44 & 0.19 \\
\hline $\operatorname{Var}\left(\eta_{4}\right)$ & 6.27 & 0.28 & Model Fit & & \\
\hline $\operatorname{Var}(\eta 5)$ & 6.24 & 0.31 & $\chi^{2}(28)$ & 87.979 & \\
\hline $\operatorname{Var}(M F)$ & 2.00 & 0.11 & RMSEA & 0.027 & \\
\hline
\end{tabular}

Note. Covariances between all latent variables are not shown but have been included. 